\title{
Optimum Power Allocation for OFDM Based Cognitive Radio Systems With Arbitrary Input Distributions
}

\author{
Ahmed Sohail, Mohammed Al-Imari, Pei Xiao, Barry G. Evans \\ Centre for Communication Systems Research \\ University of Surrey \\ Guildford, GU2 7XH, United Kingdom \\ Email: \{a.sohail,m.al-imari,p.xiao,b.evans\}@ surrey.ac.uk
}

\begin{abstract}
In the literature, optimal power assuming Gaussian input has been evaluated in OFDM based Cognitive Radio (CR) systems to maximize the capacity of the secondary user while keeping the interference introduced to the primary user band within tolerable range. However, the Gaussian input assumption is not practical and Finite Symbol Alphabet (FSA) input distributions, i.e., M-QAM are used in practical systems. In this paper, we consider the power optimization problem under the condition of FSA inputs as used in practical systems, and derive an optimal power allocation strategy by capitalizing on the relationship between mutual information and minimum mean square error. The proposed scheme is shown to save transmit power in a CR system compared to its conventional counterpart, that assumes Gaussian input. In addition to extra allocated power, i.e., power wastage, the conventional power allocation scheme also causes nulling of more subcarriers, leading to reduced transmission rate, compared to the proposed scheme. The proposed optimal power algorithm is evaluated and compared with the conventional algorithm assuming Gaussian input through simulations. Numerical results reveal that for interference threshold values ranging between $1 \mathrm{~mW}$ to $3 \mathrm{~mW}$, the transmit power saving with the proposed algorithm is in the range between $55-75 \%$, $42-62 \%$ and $12-28 \%$ whereas the rate gain is in the range between $16.8-12.4 \%, 13-11.8 \%$ and $3-5.8 \%$ for BPSK, QPSK and 16-QAM inputs, respectively.

Index Terms-Cognitive Radio, OFDM, Finite Symbol Alpha-
\end{abstract} bet, MMSE, Mutual Information.

\section{INTRODUCTION}

The rapid development of modern communication services results in high data rate requirements from the end user, which is challenging to meet because of prevailing issues such as spectrum scarcity and spectrum underutilization due to the fixed spectrum assignment policy. However, studies conducted by Ofcom [1] and FCC [2] clearly suggest that spectrum scarcity is mainly due to the inflexible spectrum licensing scheme rather than physical spectrum shortage. Cognitive Radio (CR), being the enabler of dynamic spectrum management techniques, has been proposed to overcome the spectrum underutilization problem by allowing opportunistic access of the licensed frequency band by the Secondary User (SU) under the condition of acceptable interference to the Primary User (PU) band [3]. The following spectrum sharing schemes have been presented for CR systems in [4], e.g., Underlay Spectrum
Sharing (USS), Overlay Spectrum Sharing (OSS) and Interweave (opportunistic) Spectrum Sharing (ISS) schemes. The ISS scheme is preferable due to its ability to achieve higher data rates as it allows the SU to opportunistically access the PU band.

In current wireless communication standards and services, Orthogonal Frequency Division Multiplexing (OFDM) is widely used due to its mitigation of multipath [5]. It is also very suitable for $\mathrm{CR}$ systems because it has the capability to monitor the PU spectral activity and flexibility to dynamically allocate unused licensed spectrum among SU subcarriers [6].

In opportunistic spectrum access where PU and SU co-exist in adjacent bands, mutual interference is the limiting factor on performance of both networks. Power allocation in OFDM based CR systems aims to dynamically control the transmit power on each subcarrier of the SU in order to reduce mutual interference. Therefore traditional power allocation schemes, i.e., water-filling etc. will cause more interference in the cognitive scenario, hence, a judicious power loading scheme is required which should take into consideration the fading gain of the subcarrier as well as spectral distance between the subcarriers and the PU's band. In the literature, an optimal and ladder based suboptimal power profile was proposed in [7] based on the position of the SU with respect to the PU. The same optimal power allocation scheme has been evaluated in Filter Bank Multicarrier (FBMC) based CR systems in [8], where authors claimed that FBMC achieves higher data rates due to its improved signalling shape compared to OFDM but at the cost of higher complexity. In [9], authors have evaluated optimal power in the uplink scenario for OFDM and FBMC based CR systems. However, it has been assumed that the SU transmission will continue until its completion. This imposes a requirement on the reliable transmission of subcarriers in order to guarantee a certain QoS. In [10] and [11], authors have derived optimal power by allocating more power to the subcarriers that are more frequently available for SU transmission in comparison to those active due to the PU activity.

The major drawback of the aforementioned schemes is that, researchers have optimized the transmit power assuming 


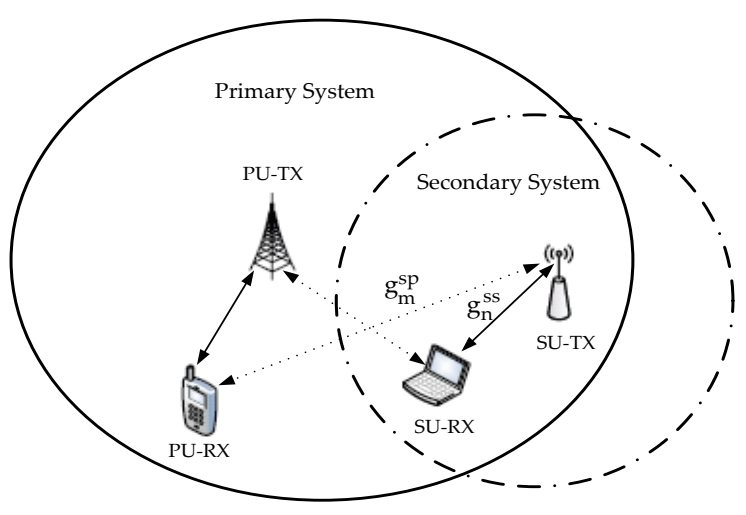

Fig. 1. Distribution of PU and SU.

Gaussian input to maximize the SU data rate for a given interference threshold value. However, Gaussian input distribution is not a valid assumption for practical systems, hence, Finite Symbol Alphabet (FSA) input distribution, i.e., MQAM is used in practical systems. The focus of this study is to optimize the transmit power for the SU while taking into account constraints imposed by practical systems, such as FSA inputs. In [12], a mercury water-filling algorithm is evaluated in order to derive optimal power allocation using the FSA input. However in this work, authors considered a non-cognitive scenario, whereas in CR systems the mercury water-filling algorithm cannot be applied due to mutual interference which limits the performance of both PU and SU networks. To address this, we propose to formulate a convex optimization problem and derive optimal power allocation for FSA input by capitalizing on the relationship between MI and Minimum Mean Square Error (MMSE) [13]. We show that if conventionally optimized power under Gaussian input assumption is used for FSA transmission, there is a wastage of transmit power; whereas the optimal power allocation derived by the proposed scheme leads to a power saving. Furthermore, the conventional scheme also results in a reduced transmission rate due to the fact that extra allocated power causes nulling of more subcarriers compared to the proposed scheme.

The remainder of the paper is organized as follows. Sections III and IV present the system model and optimal power allocation policy, respectively. We present the simulation results of the proposed scheme in Section V. Finally, conclusions are drawn in Section VI.

\section{SYSTEM MODEL}

The system model consists of a single-cell wireless system in the downlink path, where the PU and the SU transceivers coexist in the same geographical location as shown in Fig. 1. The instantaneous fading gains considered in the simulations are listed as follows, and are assumed to be known a-priori at the SU transmitter via a pilot-assisted channel estimation

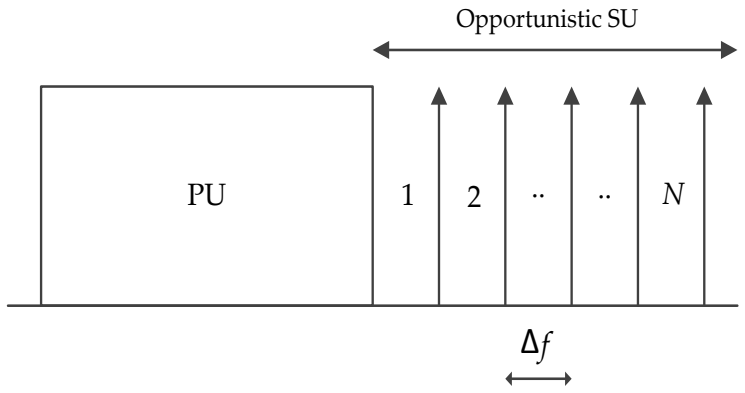

Fig. 2. Co-existence of PU and SU in frequency localized way.

algorithm: (i) $g_{n}^{s s}$, between the $\mathrm{SU}$ transmitter and SU receiver for the $n$th subcarrier; and (ii) $g_{m}^{s p}$, between the SU transmitter and $m$ th PU receiver. It has been assumed that the SU employs OFDM modulation scheme for transmission, where the available bandwidth is divided into $N$ subcarriers separated by $\Delta f$ bandwidth. As shown in Fig. 2, we consider the co-existence of primary and secondary users in a frequency domain where the discrete Fourier transform outputs are mapped to consecutive subcarriers.

In the CR system, the transmit power and achievable data rate of the SU are limited by the interference threshold imposed by the PU. Therefore, we propose to calculate an optimal power with FSA input distributions based on the convex optimization problem. The derived optimal power will be dynamically allocated to each SU subcarrier for a given channel fading gain such that the total transmission rate of the $\mathrm{SU}$ is maximized while keeping the interference introduced into the PU band within a certain threshold level. The MI is given as in [13]

$$
I_{\left(s_{i}\right)}(s n r)=\int_{0}^{s n r} m m s e(\gamma) d \gamma . \quad \text { (bits/channel use) }
$$

In this equation, $s_{i}$ denotes the arbitrary input distributions, e.g., BPSK, QPSK, 16-QAM or Gaussian etc. MMSE as presented in [12] is given by considering an $M$-ary modulation defined by $M$ discrete points denoted by $\left\{s_{l}\right\}_{l=1}^{M}$, taken with probabilities $\left\{q_{l}\right\}_{l=1}^{M}$ such that $\sum_{l=1}^{M} q_{l}=1$

$$
\begin{aligned}
\operatorname{mmse}_{\left(s_{i}\right)}( & \text { snr }) \\
& =1-\frac{1}{\pi} \int \frac{\left|\sum_{l=1}^{M} q_{l} s_{l} e^{-\left|y-\sqrt{s n r} s_{l}\right|^{2}}\right|^{2}}{\sum_{l=1}^{M} q_{l} e^{-\left|y-\sqrt{s n r} s_{l}\right|^{2}}} d y,
\end{aligned}
$$

where the integral is over the complex field. The total MI is the sum of the MI of $N$ available subcarriers calculated as follows

$$
I_{\left(s_{i}\right) \text { Total }}=\sum_{n=1}^{N} I_{\left(s_{i}\right)}(s n r) .
$$

In the ISS scheme, due to adjacent co-existence of PU and SU, two types of interference have been introduced in the system. 
One is introduced from the PU into the SU band, and the other is introduced from the SU into the PU band. Our objective is to protect the PU from unacceptable interference, therefore, in this paper we will consider interference introduced by the SU into the PU band.

\section{A. Interference introduced by the secondary user's signal}

The power density spectrum of the $n$th subcarrier in the SU band can be written as [7]

$$
\phi_{n}(f)=p_{n} T_{s}\left(\frac{\sin \pi f T_{s}}{\pi f T_{s}}\right)^{2},
$$

where $p_{n}$ is the transmit power of the $n$th subcarrier in the secondary user's band and $T_{s}$ is the symbol duration. The interference introduced by the $n$th subcarrier to the PU band is the integration of the power density spectrum of the $n$th subcarrier across the PU band and can be written as

$$
J_{n}\left(d_{n}, p_{n}\right)=g_{m}^{s p} p_{n} T_{s} \int_{\left(d_{n}-\frac{1}{2}\right) \Delta f}^{\left(d_{n}+\frac{1}{2}\right) \Delta f}\left(\frac{\sin \pi f T_{s}}{\pi f T_{s}}\right)^{2} d f,
$$

where $d_{n}$ represents the spectral distance between the $n$th subcarrier of SU and the PU band. $J_{n}\left(d_{n}, p_{n}\right)$ represents the interference introduced by the $n$th subcarrier of the SU into the PU band. The interference Eq. (5) also takes into account channel gain from the SU base station to the PU receiver, i.e., $g_{m}^{s p}$. Without loss of generality, we use a normalized channel gain of 1 .

\section{Optimal Power Allocation Policy}

The objective is to calculate an optimal power with arbitrary input distribution that maximizes the achievable data rate of the SU provided that the interference introduced into the PUs' band does not exceed a certain level. This problem can be defined as an optimization problem as follows

$$
\max _{p_{n}} \sum_{n=1}^{N} I_{\left(s_{i}\right)}\left(p_{n} g_{n}^{s s}\right),
$$

subject to

$$
\begin{gathered}
\sum_{n=1}^{N} J_{n}\left(d_{n}, p_{n}\right)=\tau_{t h}, \\
p_{n} \geq 0 \quad \forall n=1,2, \cdots, N,
\end{gathered}
$$

where $\tau_{t h}$ denotes the interference threshold prescribed by the PU. The relationship between MI and MMSE is the key to solve the optimum power allocation problem and is given by [13]

$$
\frac{d I_{\left(s_{i}\right)}(s n r)}{d(s n r)}=\operatorname{mmse}_{\left(s_{i}\right)}(s n r) .
$$

Theorem: Optimal power with arbitrary input distribution that maximizes SU data rate is as follows

$$
p_{n}^{\star}= \begin{cases}\frac{1}{g_{n}^{s s}} \operatorname{mmse}_{\left(s_{i}\right)}^{-1}\left(\frac{\lambda k_{n}}{g_{n}^{s s}}\right) & \text { if } \frac{g_{n}^{s s}}{k_{n}}>\lambda, \\ 0 & \text { if } \frac{g_{n}^{s s}}{k_{n}} \leq \lambda,\end{cases}
$$

where $\lambda$ is the Lagrange multiplier which can be calculated using numerical methods (such as bisection, secant, or Newton) for solving the following equation:

$$
\sum_{n=1}^{\left(N, \frac{g_{n}^{s s}}{k_{n}}>\lambda\right)} \frac{1}{g_{n}^{s s}} \operatorname{mmse}_{\left(s_{i}\right)}^{-1}\left(\frac{\lambda k_{n}}{g_{n}^{s s}}\right) k_{n}-\tau_{t h}=0 .
$$

Proof: As the MI is concave [14, section 2.7], the objective function in Eq. (6) is also concave because the summation preserves concave function. Also, the constraints in Eq. (7) and Eq. (8) are linear functions of the power. Consequently, the optimization problem is convex [15]. The Slater condition is satisfied with any positive power, $p_{n}>0$, that satisfy the interference constraint. Therefore, the Karush-Kuhn-Tucker (KKT) conditions are necessary and sufficient for the optimal solution. The Lagrangian for the primal problem is as follows

$$
\begin{aligned}
& L(\mathbf{p}, \lambda, \boldsymbol{\nu})=-\sum_{n=1}^{N} I_{\left(s_{i}\right)}\left(p_{n} g_{n}^{s s}\right)+\lambda\left(\sum_{n=1}^{N} J_{n}\left(d_{n}, p_{n}\right)-\tau_{t h}\right) \\
& -\nu_{n} p_{n} .
\end{aligned}
$$

The KKT conditions are as follows

Gradient of Lagrangian with respect to $p_{n}^{\star}$ vanishes

$$
-\frac{\partial I_{\left(s_{i}\right)}\left(p_{n}^{\star} g_{n}^{s s}\right)}{\partial p_{n}}+\lambda \underbrace{\frac{\partial J_{n}}{\partial p_{n}}}_{K_{n}}-\nu_{n}=0,
$$

where $k_{n}=\frac{\partial J_{n}}{\partial p_{n}}=T_{s} \int_{\left(d_{n}-\frac{1}{2}\right) \Delta f}^{\left(d_{n}+\frac{1}{2}\right) \Delta f}\left(\frac{\sin \pi f T_{s}}{\pi f T_{s}}\right)^{2} d f$,

$$
\begin{gathered}
\nu_{n} \geq 0, \quad p_{n}^{\star} \geq 0, \quad \lambda \geq 0, \\
\nu_{n} p_{n}^{\star}=0 .
\end{gathered}
$$

Using the fact that $\frac{\partial I_{\left(s_{i}\right)}\left(p_{n} g_{n}^{s s}\right)}{\partial p_{n}}=g_{n}^{s s} \operatorname{mmse}_{\left(s_{i}\right)}\left(p_{n} g_{n}^{s s}\right)$, Eq. (13) can be rewritten as

$$
-g_{n}^{s s} \operatorname{mmse}_{\left(s_{i}\right)}\left(p_{n}^{\star} g_{n}^{s s}\right)+\lambda k_{n}-\nu_{n}=0 .
$$

From Eq. (14) and Eq. (16) we have

$$
\frac{g_{n}^{s s}}{k_{n}} \operatorname{mmse}_{\left(s_{i}\right)}\left(p_{n}^{\star} g_{n}^{s s}\right) \leq \lambda,
$$

and from Eq. (15) and Eq. (16) we obtain

$$
p_{n}\left\{\lambda k_{n}-g_{n}^{s s} \operatorname{mmse}_{\left(s_{i}\right)}\left(p_{n}^{\star} g_{n}^{s s}\right)\right\}=0 .
$$

Consequently, if $p_{n}^{\star}>0$ then from Eq. (18) we have $\lambda=\frac{g_{n}^{s s}}{k_{n}} \operatorname{mmse}_{\left(s_{i}\right)}\left(p_{n}^{\star} g_{n}^{s s}\right)$, therefore

$$
p_{n}^{\star}=\frac{1}{g_{n}^{s s}} \operatorname{mmse}_{\left(s_{i}\right)}^{-1}\left(\frac{\lambda k_{n}}{g_{n}^{s s}}\right) .
$$

Since $\operatorname{mmse}_{\left(s_{i}\right)}\left(p_{n}^{\star} g_{n}^{s s}\right)<1$ when $p_{n}^{\star}>0$, we obtain from Eq. (17) $\frac{g_{n}^{s s}}{k_{n}}>\lambda$. On the other hand, as the $\operatorname{mmse}(0)=1$, if $p_{n}^{\star}=0$, we have from Eq. (17) $\frac{g_{n}^{s s}}{k_{n}} \leq \lambda$.

Note that in [7], optimal power is derived only for Gaussian input, whereas, our optimal power derivation is generic and is valid for any input distribution. It is worth mentioning that the structure of our proposed optimal power scheme is similar to the mercury water-filling scheme in [12]. 


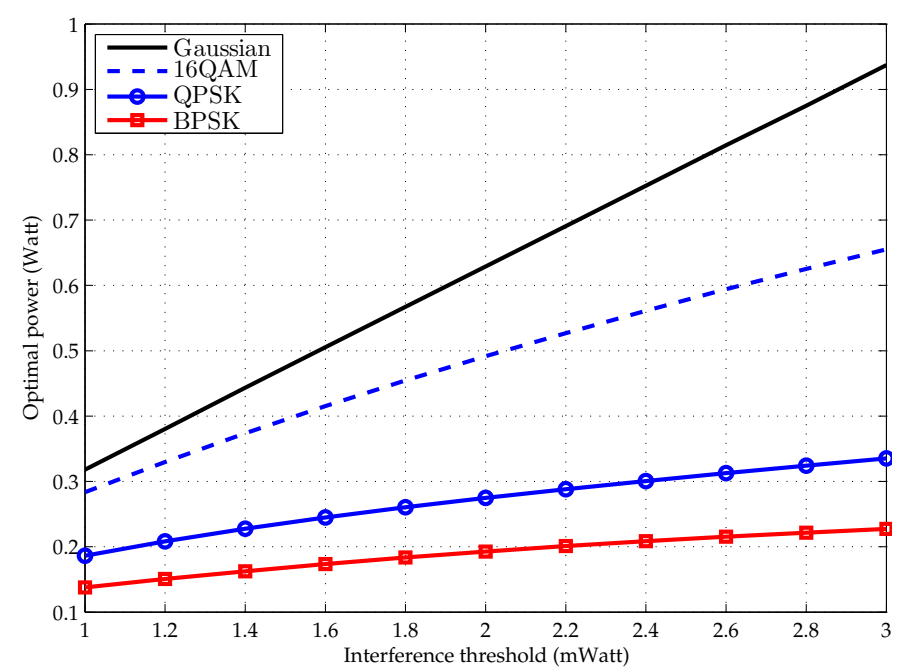

Fig. 3. Optimal power under Gaussian and FSA inputs vs $\tau_{t h}$.

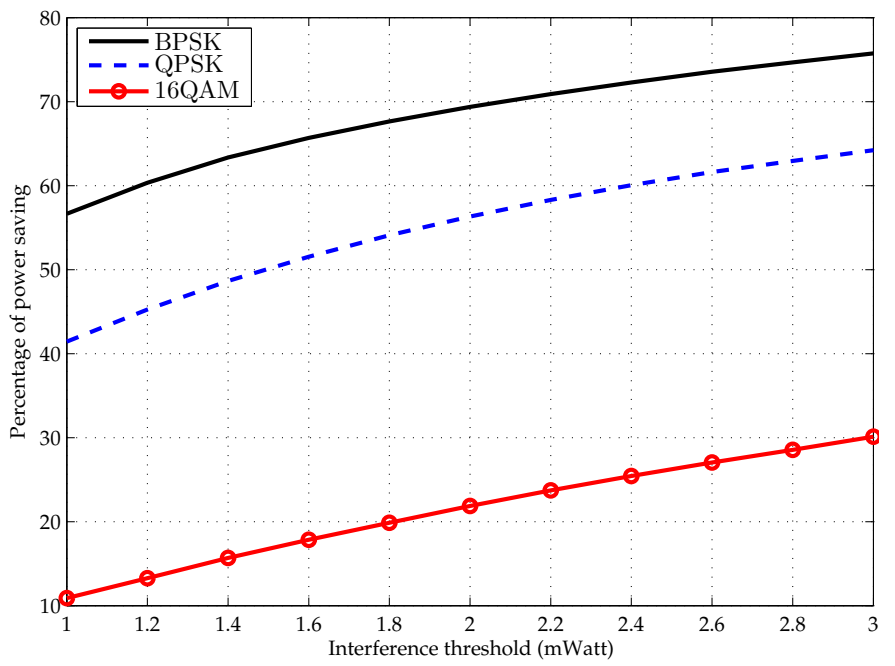

Fig. 4. Percentage of power saving vs $\tau_{t h}$.

\section{EVAluation OF OFDM BASEd CR System}

In this section, we compare optimal power and achievable data rate for Gaussian and FSA inputs in CR networks. The simulations are performed for a Single Input Single Output (SISO)-OFDM based CR network via an opportunistic scheme. Consider that there are 32 subcarriers available for the $\mathrm{SU}$ transmission in a frequency localized manner as shown in Fig. 2. The values of $T_{s}, \Delta f$ are $4 \mu \mathrm{s}, 0.3125 \mathrm{MHz}$ whereas $\tau_{\text {th }}$ ranges from 1 to $3 \mathrm{~mW}$, respectively. We further assume the IEEE 802.11 multipath channel model with root mean square delay spread of $50 \mathrm{~ns}$. The results are averaged over 2000 snapshots. We represent total transmit optimal power $\left(P^{\star}=\sum_{n=1}^{N} p_{n}^{\star}\right)$ with Gaussian input as $P_{g}^{\star}$ and with FSA input, i.e., BPSK, QPSK, 16-QAM etc. as $P_{F S A}^{\star}$.

In Fig. 3, we compare $P_{g}^{\star}$ and $P_{F S A}^{\star}$ versus $\tau_{t h}$. We observe from this figure that $P_{g}^{\star}$ is always greater than $P_{F S A}^{\star}$ over the entire $\tau_{t h}$ range. However, the power difference gap is smaller at lower $\tau_{t h}$ values as compared to higher $\tau_{t h}$ values. The

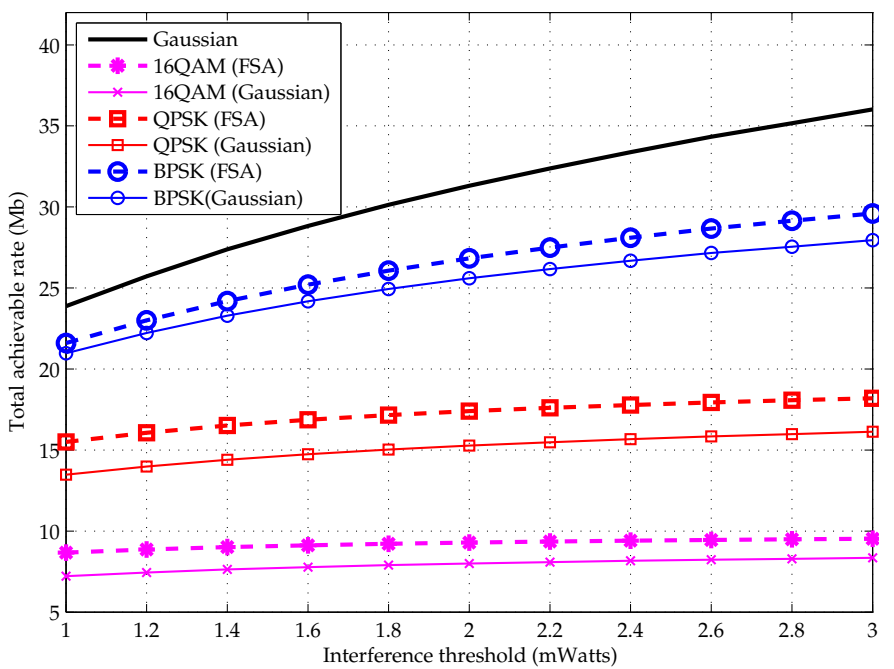

Fig. 5. Achievable data rate under Gaussian and FSA inputs vs $\tau_{t h}$.

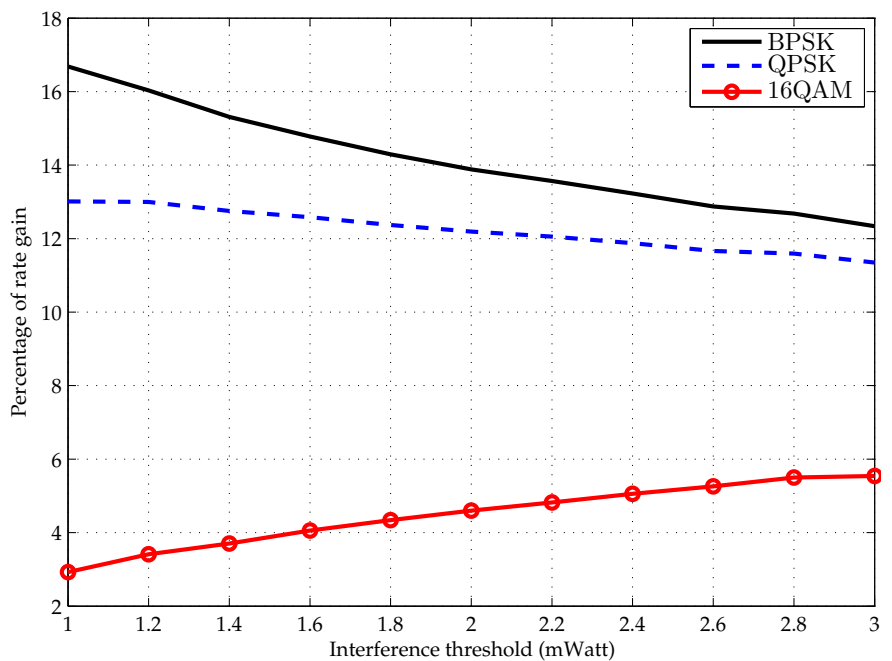

Fig. 6. Percentage of rate gain vs $\tau_{t h}$.

reasons for the power discrepancies are: (i) the increase in $P_{F S A}^{\star}$ is marginal at high $\tau_{t h}$ values because the rate reaches an upper bound limit, i.e., $\log _{2}|\mathbb{F}|$, where $\mathbb{F}$ denotes FSA set and |.| represent cardinality of the set; (ii) on the other hand, $P_{g}^{\star}$ increases with increasing $\tau_{t h}$ values because the rate under $P_{g}^{\star}$ has no upper bound limit. It is also observed that, with the same interference threshold value, $P_{F S A}^{\star}$ increases with increasing modulation scheme, i.e., from BPSK to MQAM. Therefore, after deriving optimal power for higher modulation schemes, (i.e., 16-QAM) and using the lower modulation scheme, (i.e., BPSK) for transmission results in waste of transmit power. Hence, for efficient power utilization, power must be optimized according to the actual employed modulation scheme.

We demonstrate the power saving by using our proposed power allocation scheme $\left(P_{F S A}^{\star}\right)$ comparing to Gaussian in Fig. 4, where we plot percentage power saving $=\frac{P_{g}^{\star}-P_{F S A}^{\star}}{P_{t}^{\star}} 100$ for BPSK, QPSK and 16-QAM versus $\tau_{t h}$, respectively. From 


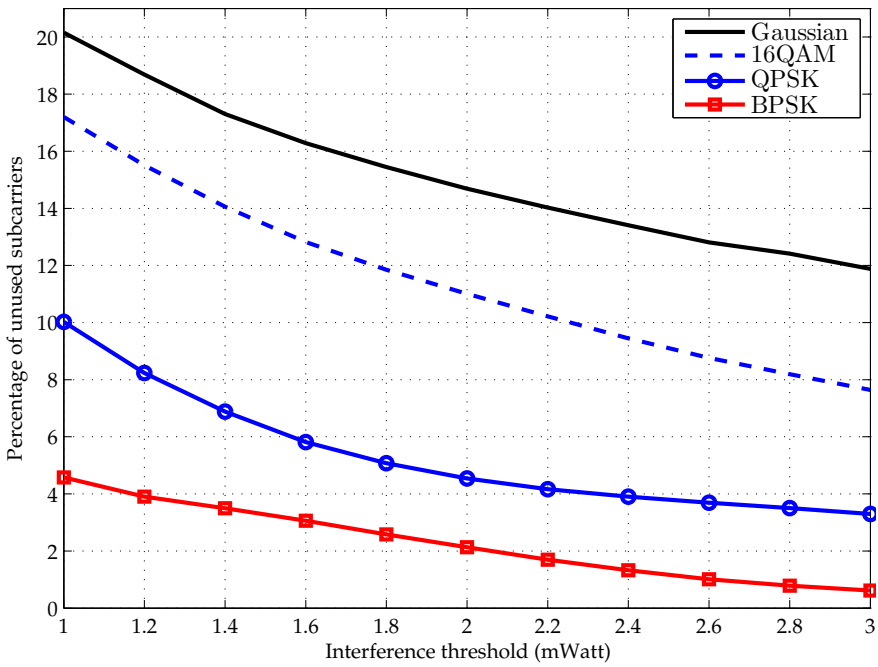

Fig. 7. Percentage unused subcarriers vs $\tau_{t h}$.

this figure, we observe that there is significant power saving by using the proposed optimal power $P_{F S A}^{\star}$ compared to $P_{g}^{\star}$. For $\tau_{t h}$ values ranging from $1 \mathrm{~mW}$ to $3 \mathrm{~mW}$, the transmit power saving is $55-75 \%, 42-62 \%$ and $12-28 \%$ with BPSK, QPSK and 16-QAM inputs, respectively.

Fig. 5 shows a comparison of achieved data rate for FSA transmission between power optimized for Gaussian input and the case when the power is optimized based on the actual modulation scheme. The figure shows that the proposed optimal power allocation scheme achieves higher data rate compared to the traditional Gaussian power allocation scheme. To elaborate, Fig. 6 shows percentage rate gain versus $\tau_{t h}$ values ranging from $1 \mathrm{~mW}$ to $3 \mathrm{~mW}$; the rate gain is $16.8-12.4 \%$, $13-11.8 \%$ and $3-5.8 \%$ for BPSK, QPSK and 16-QAM inputs, respectively. The justification for this is that in the cognitive radio scheme where primary and secondary users coexist in adjacent bands, the SU subcarriers which are closer to the PU band cause higher interference, therefore, less or even zero power is allocated to these subcarriers. As $P_{g}^{\star}$ is always higher than $P_{F S A}^{\star}$, it nulls more subcarriers compared to optimum power under the FSA input and ultimately these subcarriers will be wasted. Fig. 7 depicts the unused subcarriers among various schemes, i.e., BPSK, QPSK, 16-QAM and Gaussian versus $\tau_{t h}$. For example, for the $1 \mathrm{~mW}$ case, $P_{g}^{\star}$ causes five additional nulling subcarriers compared to $P_{B P S K}^{\star}$, resulting in $16.8 \%$ rate loss.

\section{CONCLUSiON}

In this paper, we have considered the power allocation problem in cognitive radio systems under the condition of FSA inputs as in practical systems. Using standard convex optimization techniques, the optimal power allocation is derived by capitalizing on the relationship between MI and MMSE. The derived optimal solution under the FSA is evaluated and compared with its conventional counterpart that assumes Gaussian input. Numerical results have shown that the proposed scheme outperforms the power allocation based on Gaussian inputs in terms of transmit power saving and achieved data rate. Consequently, system spectrum efficiency and energy efficiency can be improved by using the proposed power allocation scheme. Furthermore, we have shown that as the modulation order increases, the optimal transmit power also increases. Therefore, the power should be optimized based on the actual used modulation scheme to achieve a desired energy efficiency.

\section{ACKNOWLEDGMENT}

This work has been supported by the India UK Advance Technology Center of Excellence in Next Generation Networks, Systems and Services (www.iu-atc.com).

\section{REFERENCES}

[1] A. Shukla, "Cognitive radio technology-a study for Ofcom," Tech.Rep. 830000143, QinetiQ Ltd, Hampshire, UK, 2006.

[2] "Federal communications commission: Spectrum policy task force report," http://fjallfoss.fcc.gov, nov. 2002.

[3] A. Sendonaris, E. Erkip, and B. Aazhang, "User cooperation diversity. part I. system description," IEEE Transactions on Communications, vol. 51, no. 11, pp. 1927-1938, Nov. 2003.

[4] S. Srinivasa and S. Jafar, "Cognitive radios for dynamic spectrum access - the throughput potential of cognitive radio: A theoretical perspective," IEEE Communications Magazine, vol. 45, no. 5, pp. 73-79, May 2007.

[5] H. Mahmoud, T. Yucek, and H. Arslan, "OFDM for cognitive radio: merits and challenges," IEEE Wireless Communications, vol. 16, no. 2, pp. 6-15, April 2009.

[6] T. Weiss, J. Hillenbrand, A. Krohn, and F. Jondral, "Mutual interference in OFDM-based spectrum pooling systems," in 59th IEEE Vehicular Technology Conference, 2004, vol. 4, May 2004, pp. 1873 - 1877 Vol.4.

[7] G. Bansal, M. Hossain, and V. Bhargava, "Optimal and suboptimal power allocation schemes for OFDM-based cognitive radio systems," IEEE Transactions on Wireless Communications, vol. 7, no. 11, pp. 4710-4718, Nov. 2008

[8] M. Shaat and F. Bader, "Low complexity power loading scheme in cognitive radio networks: FBMC capability," in The 20th IEEE Personal, Indoor and Mobile Radio Communications Symposium, Sept. 2009, pp. 2597-2602.

[9] — "A two-step resource allocation algorithm in multicarrier based cognitive radio systems," in IEEE Wireless Communications and Networking Conference, April 2010, pp. 1-6.

[10] Z. Hasan, G. Bansal, E. Hossain, and V. Bhargava, "Energy-efficient power allocation in OFDM-based cognitive radio systems: A risk-return model," IEEE Transactions on Wireless Communications, vol. 8, no. 12, pp. 6078-6088, Dec. 2009.

[11] C. Chiuan-Hsu and W. Chin-Liang, "Power allocation for ofdm-based cognitive radio systems under primary user activity," in IEEE 71st Vehicular Technology Conference, May 2010, pp. 1-5.

[12] A. Lozano, A. Tulino, and S. Verdu, "Optimum power allocation for parallel gaussian channels with arbitrary input distributions," IEEE Transactions on Information Theory, vol. 52, no. 7, pp. 3033-3051, July 2006.

[13] D. Guo, S. Shamai, and S. Verdu, "Mutual information and minimum mean-square error in gaussian channels," IEEE Transactions on Information Theory, vol. 51, no. 4, pp. 1261-1282, April 2005.

[14] T. M. Cover and J. A. Thomas, Elements of Information Theory-2nd Edition. Hoboken, New Jersey: Wiley, 2006.

[15] Z.-Q. Luo and W. Yu, "An introduction to convex optimization for communications and signal processing," IEEE Journal on Selected Areas in Communications, vol. 24, no. 8, pp. 1426-1438, Aug. 2006. 